\title{
Religion als Hemmnis und Medium lokaler Integration
}

Hansjörg Schmid und Claus Leggewie

\section{Zusammenfassung}

Religiöse Pluralität ist heute ein unverkennbarer und irreversibler Faktor, den jede Integrationspolitik in Rechnung stellen muss. Während die Gesellschaft weitgehend von Säkularisierungsprozessen geprägt ist, gewinnt gerade im Zusammenhang mit Migration Religion für viele Menschen an Bedeutung. Religion wirkt einerseits stabilisierend und integrationsförderlich, erzeugt oder verschärft aber andererseits auch Konflikte, indem transnationale oder globale Auseinandersetzungen auf die kommunale Ebene einwirken und religiös-normative Deutungen soziale Konfliktkonstellationen zusätzlich aufladen. Lokaler Integrationspolitik kommt hier die Aufgabe einer Konfliktmoderation zu. Sie kann durch die Einbeziehung neuer, noch nicht etablierter religiöser Akteure deren Potenziale nutzen und so zu einem friedlichen Zusammenleben beitragen. Dabei ist erforderlich, auch die spezifischen Strukturen und Positionen der jeweiligen Religionen in ihrer Vielfalt zu berücksichtigen. In Deutschland sind das vor allem Muslime, in einem zahlenmäßig geringeren Umfang aber auch Juden sowie Angehörige christlicher Freikirchen, christlich-orthodoxer Gemeinden und weiterer Religionen. Auch wenn die öffentlichen Debatten, bisherige wissenschaftliche Untersuchungen und damit auch folgenden Überlegungen stark auf den Islam fokussiert sind, ist es wichtig, die genannten weiteren Akteure ebenso in den Blick zu nehmen, auf die sich vieles analog übertragen lässt.

\section{Religiöse Vielfalt - multireligiöse Städte und Gemeinden}

Deutschland ist wie die meisten anderen europäischen Länder auch von einer wachsenden religiösen Vielfalt geprägt (Klöcker / Tworuschka 1997ff.). Wie die religiöse Diversität seit dem Zweiten Weltkrieg zugenommen hat, zeigt beispielhaft ein Vergleich (Pollack / Müller 2013, 32f.): Während 1950 in Gesamtdeutschland über 95 Prozent einer der beiden großen Kirchen angehört haben, sind das 2010 nur noch rund 60 Prozent, davon 30,2 Prozent Katholiken und 29,2 Prozent Protestanten. 30,3 Prozent sind konfessionslos, die restlichen 
rund zehn Prozent verteilen sich auf verschiedene Bekenntnisse, darunter als größte Gruppe Muslime mit rund fünf Prozent. Es folgen Angehörige der unterschiedlichsten Freikirchen und orthodoxen Kirchen, Buddhisten, Hinduisten und Juden. Abbildung 1 demonstriert anhand des Beispiels von Nordrhein-Westfalen den hohen religiösen Diversitätsgrad ${ }^{1}$ gerade in den Ballungsgebieten an Rhein und Ruhr (Hero et al. 2008, 222):

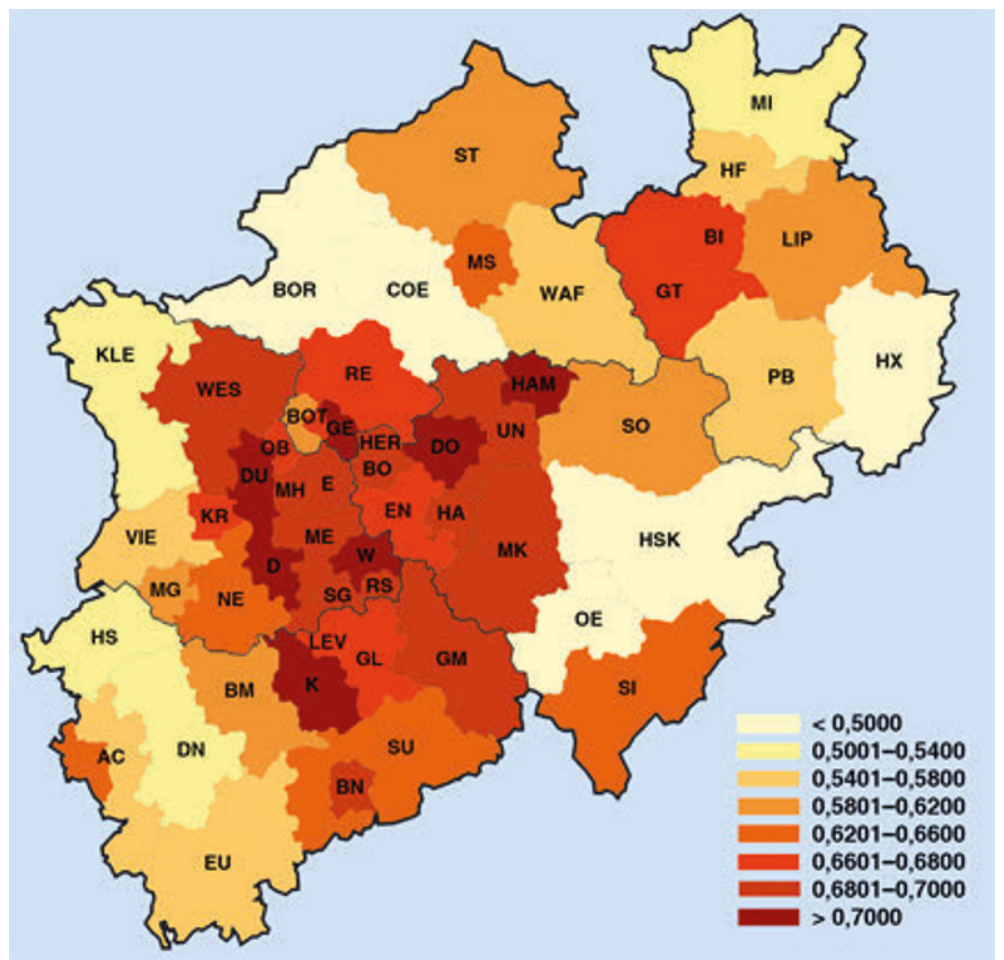

Abb. 1 Religiöse Vielfalt im Bundesland Nordrhein-Westfalen

Quelle: http://plureligion.net/en/mapping-religious-diversity/northrhine-westphalia/diversity-maps/ (Diversity of Affiliation)

Abbildung 2 stellt den Anteil von Zuwanderinnen und Zuwanderern ohne deutschen Pass dar (Hero et al. 2008, 219). Es illustriert, wie Diversitätsgrad und Migrationsdichte korrelieren.

1 Bei Diversitätsindex 0 bestünde ein Monopol einer Religionsgemeinschaft; je höher der Diversitätsindex gegen 1 tendiert, desto diverser ist die religiöse Lage. 


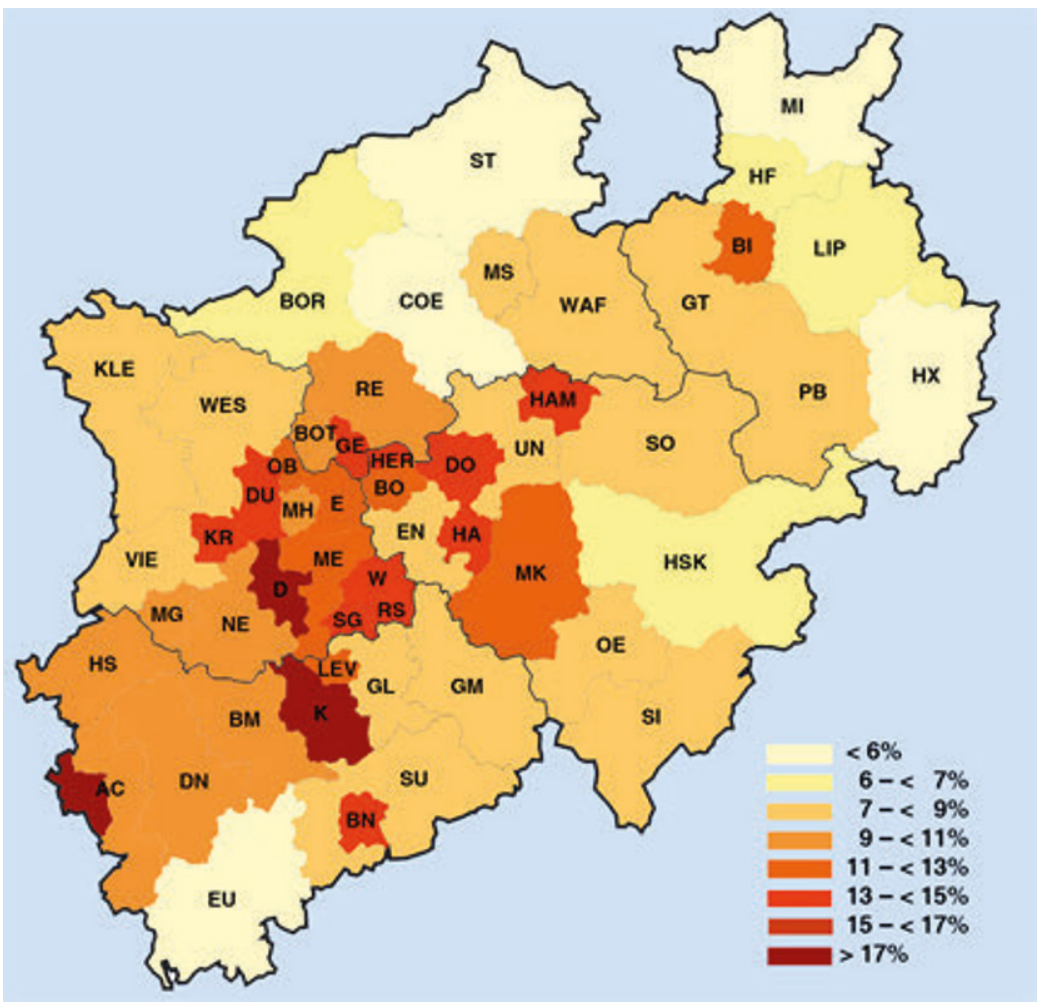

Abb. 2 Religiöse Vielfalt durch Einwanderung

Quelle: http://plureligion.net/en/mapping-religious-diversity/northrhine-westphalia/diversity-maps/ (Migration Density)

Wichtig ist dabei auch der negative Zusammenhang zwischen Diversität und Organisation: Je vielfältiger eine Religionslandschaft ist, desto weniger sind Gläubige in kirchlichen und Gemeindeorganisationen konzentriert (Krech 2008, 35f.). Diese Ergebnisse zu Nordrhein-Westfalen decken sich grosso modo mit Daten aus der übrigen (alten) Bundesrepublik, die im Süden und Norden etwas homogener ist, während die neuen Bundesländer generell stärker durchsäkularisiert sind, aber stellenweise auch offener für freikirchliche Missionierung. Während also die Bindungskraft etablierter religiöser Organisationen vor allem in den Großstädten und Ballungsgebieten abnimmt, steigt gerade dort die Intensität religiöser und religionsbezogener Kommunikation. Und die Bedeutung der Religion ist bei Muslimen generell höher als bei Angehörigen anderer Religionsgemeinschaften; dies gilt auch bei hohem formalem Bildungsgrad. 


\section{Religiöse Rekodierung und Renaissance}

Bestätigt wird dieses Bild dadurch, dass ,Hochreligiöse‘ aller Glaubensrichtungen, für die besonders intensiv erlebte religiöse Überzeugungen eine zentrale Rolle spielen und das gesamte, auch politische Verhalten leiten, im religiösen und öffentlichen Diskurs eine zentrale Rolle spielen (Bertelsmann Stiftung 2007). Eine besondere Facette davon ist die hohe Bedeutung der Religion und der Religiosität für in Deutschland lebende Muslime, die auf eine Rekodierung der Minderheitenexistenz in der Fremd- und Selbstwahrnehmung hindeutet (Brettfeld / Wetzels 2009, 242ff.; Pollack / Müller 2013, 17). So halten sich nach der repräsentativen Studie „Muslimisches Leben in Deutschland“ auf einer vierteiligen Skala 36 Prozent der Muslime für „sehr stark gläubig“ und 50 Prozent für „eher gläubig“ (Haug et al. 2009, 139). Allerdings erweisen sich nicht-muslimische Migranten aus den entsprechenden Herkunftsregionen ähnlich stark religiös.

Was die Fremdwahrnehmung betrifft, werden Minderheiten seit Ende der 1970er Jahre stärker im Hinblick auf ihre ethnisch-religiöse Identität gekennzeichnet. Gerade im Hinblick auf Immigranten türkischer Abstammung, der größten Einwandererpopulation in Deutschland, kann man sagen, dass im Zeitraum zwischen 1960 und heute aus eingewanderten Türken eher Kurden und Muslime wurden als Deutsche türkischer Abstammung muslimischen Glaubens (Leggewie 1993; 1996); diese äußere und/oder freiwillige Segregation hat sich nach der Reform des Staatsangehörigkeitsrechts nur noch teilweise erhalten. Laut der Studie „Muslimisches Leben in Deutschland“ empfinden 44 Prozent der Muslime einen starken, 25 Prozent einen sehr starken Bezug zu Deutschland, wohingegen nur rund die Hälfte eine starke oder sehr starke Verbundenheit mit dem Herkunftsland aufweist (Haug et al. 2009, 297). Trotzdem besteht in puncto Selbstwahrnehmung eine Verbindung zwischen einer tatsächlichen oder, gefühlten`Erfahrung kollektiver Marginalisierung mit der Radikalisierung der Ausrichtung an religiösen Regeln und Ritualen und der Übernahme fundamentalistischer Positionen. Dies gilt für Einwanderer mit islamischem Hintergrund, aber auch für evangelikale Gruppen, die sich unter ethnischen Minderheiten wachsender Beliebtheit erfreuen.

Im Blick auf lokale Konfliktkonstellationen ergibt sich daraus eine bemerkenswerte Disposition: Zum einen sind ,Hochreligiöse‘ stärker davon überzeugt, dass ihre religiöse Überzeugung richtig und diese in ,exklusiver Festigkeit' gegen Andersgläubige, Agnostiker und Atheisten zu verteidigen ist. Das kann eine gestiegene Missionsbereitschaft einschließen, also den Versuch, andere für die eigene Glaubenswahrheit zu gewinnen, aber auch das überkonfessionelle Interesse, religiöse Überzeugungen in der Öffentlichkeit zur Geltung kommen zu lassen (Huber 2007, 25). Diese Tendenz führt aber nicht notwendig zu wachsender religiöser Intoleranz. Denn zum einen geht sie vielfach einher mit religiöser Reflexivität, also der Bereitschaft, sich kritisch mit (eigenen) religiösen Dogmen und Einstellungen auseinanderzusetzen; zum anderen schließt sie den Respekt für Andersgläubige ein (Krech 2007, 36ff.).

Drei mögliche Spaltungslinien leiten sich aus diesem Gesamtbild einer ,religiösen Renaissance` $\mathrm{ab}$ : 
a. Hochreligiöse aller Glaubensrichtungen bilden eine (informell-ökumenische) Allianz und anlassspezifische Koalitionen gegen Säkulare und Atheisten.

b. Hochreligiöse setzen sich mit weniger Gläubigen im Rahmen ihrer religiösen Einrichtungen auseinander.

c. Hochreligiöse ringen mit Andersgläubigen um den Alleinvertretungsanspruch ihrer Religionsgemeinschaft.

Religiöse Vielfalt fordert also ein vorherrschendes Toleranzverständnis heraus. Toleranz bedeutet ja nicht, andere Bekenntnisse indifferent und ohne Wertung zu akzeptieren, vielmehr geht es darum, solche Bekenntnisse zu dulden, gegen die man erhebliche Einwände und Bedenken hat (Forst 2003). Man kann die Hypothese verfolgen, dass Toleranz im Sinne einer horizontal angeordneten religiösen Vielfalt umso schwieriger wird, je stärker sich die Gläubigen als Opfer sozialer und/oder politischer Unterdrückung und Diskriminierung fühlen.

\section{Religiöse Konfliktlagen: transnational und lokal}

Bevor man sich den daraus resultierenden lokalen bzw. kommunalen Konfliktlagen zuwendet, muss man diese weltgesellschaftlich einordnen. Denn ein großer Teil der religiös-politischen Selbst-Wahrnehmungen verweist auf die Relevanz und Brisanz der religiösen Frage in der kulturellen Globalisierung, die lokale Konstellationen ,überdeterminiert'. Im Prozess der Säkularisierung seit dem 17. Jahrhundert schienen religiöse Dimensionen aus den internationalen Staaten-Beziehungen verschwunden zu sein; es interessierten vornehmlich Streitigkeiten um Territorien, Rohstoffe und Minderheiten, oder allgemeiner: wirtschaftliche und strategische Ziele. Dieses Bild änderte sich mit der Zäsur 1989/1990: Der einflussreiche Politologe Samuel Huntington und andere erblicken seither allerorts und unablässig Kulturkämpfe und Religionskriege, die ökonomische und strategische Kalküle angeblich verdrängen und westliche Einwanderungsgesellschaften in Identitätskonflikte stürzen. Diese Positionen haben aber auch weitreichende Kritik erfahren (Müller 1998).

Wenigstens vier Dimensionen kann man im globalen religiösen Feld ausmachen, die die Offenheit und Ambivalenz religiös motivierter Intervention sichtbar werden lassen:

\begin{tabular}{|c|c|}
\hline Globalisierung & Religionen in Konflikten \\
Säkularisierung \& & Clash of Fundamentalisms \\
Sakralisierung & \& Friedensstiftung \\
\hline $\begin{array}{c}\text { Werte in der Außenpolitik } \\
\text { Religiöse und/oder } \\
\text { zivile Quellen }\end{array}$ & Normsetzung \\
\hline
\end{tabular}

Abb. 3 Religiöse Dimensionen internationaler Politik

(C) Claus Leggewie 
Das erste Feld ist die Politisierung der Religion im Prozess der Globalisierung, der nicht, wie man lange annahm, auf wirtschaftlich-finanzielle Transaktionen beschränkt ist, sondern seine Brisanz genau in der kulturellen und religiösen Sphäre an den Tag legt. Säkularisierung und Sakralisierung schließen sich in der Weltgesellschaft nicht aus, sie schreiten parallel voran (Norris/Inglehart 2011).

Daraus erwachsen zweitens zunehmend religiös begründete Konflikte, die mit der Ausbreitung und dem Aufeinandertreffen fundamentalistischer Strömungen oder allgemeiner ,politischer Religionen' zusammenhängen.

Drittens verweist dies auf eine alte, ebenso in säkularen Regimen feststellbare Wertorientierung von Außenpolitik, die sich meist in kaschierter Form, zum Teil aber auch ganz offen auf religiöse Quellen und Einflüsse beruft.

Schließlich ist viertens auch schon seit langem ein Beitrag religiöser Institutionen und Persönlichkeiten an der Normierung und Verrechtlichung der internationalen Beziehungen zu konstatieren.

Die große Bandbreite religiöser Motive in der internationalen Politik verwundert kaum (Leggewie 2009), stellt man den immer schon grenzüberschreitenden Charakter der großen Weltreligionen in Rechnung. Sie haben dabei den Vorzug, in der lokalen Lebenswelt geerdet zu sein und gleichwohl für die ganze Menschheit sprechen zu können. Dieser per se transnationale Grundzug der Weltreligionen, der sich in weltweit verstreuten Diaspora-Gemeinschaften niederschlägt, ist heute wieder eine Quelle von Konflikten: Nachdem der Westfälische Frieden von 1648 die Religionen exemplarisch entpolitisiert hatte und auf der friedlichen Koexistenz der Konfessionen die moderne Staatenordnung errichtete, treten nunmehr Religionsgemeinschaften , postwestfälisch ' - wieder als politische Religionen auf - gegen Staaten, neben Staaten, mit Staaten.

\begin{tabular}{|l|l|}
\hline „westfälisch“ & „postwestfälisch“ \\
\hline Friedliche Koexistenz der Konfessionen & Polytheistischer Wettbewerb \\
\hline Staat-Kirche-Allianzen & Deregulierter Religionsmarkt \\
\hline Amtskirchen und Gemeindeorganisation & Bewegungen und Netzwerke \\
\hline Religiöse Zentren und Regionen & Transnationale Diaspora \\
\hline Stabilität & Mobilität \\
\hline Versäulung & Mission/Konversion \\
\hline
\end{tabular}

Abb. 4 Von der westfälischen Staatenordnung zur postwestfälischen Weltgesellschaft (c) Claus Leggewie

Als erstes Fazit kann man festhalten, dass Religion in der Weltgesellschaft ausgesprochen vielschichtig ist und Konfliktfelder nicht nur zwischen Religion und Politik, sondern auch zwischen den religiösen Gemeinschaften und in deren Inneren zunehmen, was eine unübersichtliche Konstellation der Akteure bedingt. Religiöse Aspekte werden als Normalfall 
globaler Beziehungen und Verhältnisse erkennbar. Allerdings sind weiterhin ökonomische und politische Faktoren als zentrale Konfliktursachen anzusehen (Hasenclever 2008).

Den entscheidenden Unterschied dieser widersprüchlichen Entwicklung macht offenbar aus, ob sie unterm Strich auf Arbeitsteilung, Wettbewerb oder Konfrontation hinausläuft und wie der (im Grundsatz selbst von islamischen Republiken bekräftigte) Trennungsgrundsatz tatsächlich respektiert, also der Primat des Politischen aufrechterhalten wird. Radikal bestritten wird der in westlichen Republiken festgeschriebene Trennungsgrundsatz zum einen durch den Islamismus, über den der internationale dschihadistische Terrorismus (exemplarisch das dezentrierte Netzwerk Al-Qaida oder die Organisation „Islamischer Staat“) insofern noch hinausgeht, als er Staatenbeziehungen völlig negiert und unterläuft. Fundamentalisten aller Couleur (darunter Pfingstler und ,Wiedergeborene im protestantischen Spektrum, das die Friedensbewegungen der 1980er Jahre gegen die nukleare Aufrüstung geprägt hat) rekrutieren besonders dort, wo Staatlichkeit gescheitert ist oder posttotalitäre Vakuen mit, Sinn' zu füllen sind. Der monopolartige Bezug auf eine religiöse Identität tritt dort dezidiert an die Stelle säkular-politischer oder geographischer Selbstverortungen.

Daneben kommt auch das konfliktlindernde und friedensfördernde Potenzial der Religionen zur Geltung. Hier ist etwa an die christliche Gemeinschaft Sant'Egidio oder die muslimische Initiative „A Common Word“ zu denken (Eißler 2009). Auch sie können keinen völligen Konsens in der Gesellschaft bewirken. Konflikt ist vielmehr als Normalfall $\mathrm{zu}$ verstehen, so dass das Ziel daher nicht die völlige Eliminierung von Konflikten sein kann. Vielmehr geht es darum, sich über Mechanismen der Konfliktaustragung zu verständigen. Wichtige Schritte können der deeskalierende Ausschluss von Gewalt und anderen Konfliktmitteln, der Abbau von Ungleichheiten, das Herausarbeiten gemeinsamer Interessen, die Verständigung über normative Grundlagen und die Erarbeitung gemeinsamer Regeln (etwa der Menschenrechte) sein, deren Notwendigkeit im Konflikt erst bewusst wird. Auf diese Weise findet eine Rationalisierung von Konflikten statt. Diese bleiben bestehen als „schöpferischer Kern aller Gesellschaft“ (Ralf Dahrendorf), werden aber in einer bestimmten Form bearbeitet.

Heute wirken sich globale Phänomene und Konfliktkonstellationen stärker als früher auf die lokale Ebene aus und prägen auch die Identitäten der Menschen. Ethnisch-religiöse Konflikte in den Vorstädten, die sich aus Bildern der Intifada nähren und diese zum Teil imitieren, sind in erheblichem Umfang auf derartige globale Einflüsse zurückzuführen, die lokale Erfahrungen ,einrahmen' und radikalisieren können. Diese Tendenz ist Ausdruck der mit der kulturellen Globalisierung einhergehenden ,Glokalisierung`. ,Lokale‘ Ereignisse (wie der dänische Karikaturenstreit oder die riots in den französischen Vorstädten) gewinnen weltweite Resonanz und ziehen ihre Energie wiederum aus ,globalen', zumeist durch alte und neue elektronische Medien übermittelten Symbolereignissen.

Besonders prägend ist die mediale Wahrnehmung und Darstellung des Islams. Diese hat oft einen abgrenzenden, entdifferenzierenden und generalisierenden Charakter (Bielefeldt ${ }^{2} 2008$; Çakir 2014). Sie ist davon geprägt, dass sämtliche soziale, kulturelle oder politische Phänomene auf den Islam bezogen und allein mit seiner Hilfe gedeutet werden. Es kommt 
dadurch zu einer pauschalen, statischen und undifferenzierten Gegenüberstellung von Muslimen und Nicht-Muslimen (Kermani 2009), wobei Muslime vielfach nur unter ihrer religiösen Identität wahrgenommen und ihre multiplen Identitäten ausgeblendet werden. Dabei werden insbesondere ein hohes Gewaltpotenzial, eine Unterdrückung der Frau und eine Unfähigkeit zur Ausdifferenzierung zwischen Staat und Religion unterstellt. Verbreitet ist eine Islam- und Muslimfeindschaft, die trotz ihrer unterschiedlichen Wurzeln mit ähnlichen Stereotypen arbeitet wie Antisemitismus (Benz 2011). Daraus resultieren Exklusionen und Diskriminierungen, welche die Situation von Muslimen in Deutschland prägen (Brettfeld / Wetzels 2009, 333f.; Hafez / Schmidt 2015, 32f.).

Als Gegenbilder zum Islam werden Konstrukte wie „christliches Abendland“ oder „jüdisch-christliche Tradition“ herangezogen, um diesen als fremd und nicht zugehörig zu kennzeichnen. Dabei werden jedoch Judentum und Christentum funktionalisiert. Insbesondere eine Vereinnahmung des Jüdischen zum Zweck einer Abgrenzung gegenüber dem Islam erweist sich als problematisch, da damit nicht der Bedeutung aller drei Religionen einschließlich des Islams für die Geschichte Europas Rechnung getragen und der christlichen Antijudaismus ausgeblendet wird (Schreiner 2008). Diese Gegenbilder stellen keine geeigneten Konfliktinterventionen dar, da sie jeweils unterkomplex sind. Es handelt sich jedoch um verbreitete Identitätskonstruktionen, die sich ebenso wie die unmittelbaren Abgrenzungen gegenüber dem Islam stark auf die lokale Ebene auswirken.

\section{$4 \quad$ Lokale Konflikte und Aushandlungsprozesse}

Vor diesem Hintergrund kann man auf lokaler Ebene eine ganze Reihe religiös motivierter bzw. kodierter Konflikte konstatieren. Arenen dieser Konflikte sind öffentliche Dienste (wie Schulen, Ämter), Betriebe und Kultstätten, wo religiöse Symbole umstritten sind, aber auch Wohnquartiere, Schulhöfe und öffentliche Plätze. Je nach institutioneller und rechtlicher Flexibilität können solche Konflikte eskalieren oder vor Gerichten, im lokalen Bargaining und über die Medienöffentlichkeit ausgetragen werden. Vielfach spielen dabei gesellschaftliche Akteure wie Bürgervereine oder Initiativen auf Stadtteilebene eine zentrale Rolle. Auf staatlicher Seite tritt neben politischen Gremien, Verwaltungen und Behörden auch die Polizei als Akteur in Erscheinung. Einerseits können so Veranstaltungen etwa zur Drogenberatung und Kriminalitätsprävention durchgeführt werden; andererseits hat ihr Auftreten auch eine Versicherheitlichung der Politik zur Folge und kann bereits vorhandene Verdachtsmomente gegenüber Muslimen verstärken (Rosenow-Williams 2012, 128ff.).

Die Ausweitung des religionsrechtlichen Systems Deutschlands kann als „Inkorporationsregime“ (Soysal 1994, 29ff.) verstanden werden. Nicht nur auf Länderebene (Religionsunterricht, theologische Fakultäten), sondern auch auf kommunaler Ebene spielt dieses eine zentrale Rolle. Nachdem das religionsrechtliche System bis nach der Jahrtausendwende weitgehend auf die christlichen Kirchen (und partiell auch auf die jüdische Religionsgemeinschaft) beschränkt blieb, gibt es seitdem Bemühungen, das System zu öffnen und 
auf diese Weise etwa durch islamischen Religionsunterricht an öffentlichen Schulen oder durch islamische Theologie an Universitäten einen Beitrag zur Integration zu leisten. Dies ist aufgrund des Prinzips der Neutralität des Staates, der nicht bestimmte Religionsgemeinschaften privilegieren darf, geboten (Bielefeldt 2007, 85f.; Willems 2003, 112). Die Aufnahme in die Strukturen, die in der Regel mit bestimmten Anforderungen verknüpft ist und oft langwierige Aushandlungsprozesse nach sich zieht, führt zu wechselseitigen Veränderungen. Das Selbsthilfe-Potenzial sowie das soziale und kulturelle Kapital etwa von islamischen Organisationen kann so besser genutzt werden. Die Öffnungsprozesse haben aber auch Auswirkungen auf die etablierten kirchlichen Akteure, die sich heute nicht mehr selbstverständlich in einer privilegierten Position befinden, sondern Ansprüche und Aufgaben teilen und eine Kommunikations- und Dialogfähigkeit in verschiedene Richtungen pflegen müssen.

Auf kommunaler Ebene sind diese Arrangements vor allem im Bereich wohlfahrtsstaatlicher Handlungsfelder relevant, aber spielen auch im Blick auf Schulen, religiöse Bauten und Friedhöfe eine zentrale Rolle (Färber / Spielhaus 2006). Dabei geht es einerseits um die interkulturelle und interreligiöse Öffnung bestehender Einrichtungen, um so die Kompetenz im Umgang mit kultur- oder religionsbezogenen Konflikten zu erhöhen, andererseits um die Möglichkeit neuer Einrichtungen, die von Migrantenvereinen, Juden oder Muslimen selbst getragen werden. In der Debatte um den Aufbau eines islamischen Wohlfahrtsverbandes, der sich an der seit 1917 bestehenden Zentralwohlfahrtsstelle der Juden orientieren kann, spielt die kommunale Ebene eine Pionier- und Schlüsselrolle (Schmid 2014, 645ff.). So sind es in der Regel die Jugend- und Sozialämter auf der Ebene der Kommunen bzw. der Landkreise, die über die Anerkennung sozialer Dienste in freier Trägerschaft entscheiden.

Beim Bereich der Altenhilfe und der Seniorenarbeit handelt es sich noch weitgehend um Zukunftsthemen (Halm / Sauer 2015, 77ff.). Es gibt jedoch erste Pflegedienste und Pflegeheime, die sich besonders um religiöse und kulturelle Sensibilität bemühen. Außerdem betreiben viele muslimischen Vereine Seniorentreffs sowie Bildungs- und Beratungsangebote für Senioren. Im Zentrum steht allerdings derzeit die Kinder- und Jugendhilfe. Sie ist in Deutschland durch eine gesetzlich garantierte Trägerpluralität gekennzeichnet, die die gesellschaftliche Vielfalt widerspiegeln und Eltern wie Jugendlichen eine Wahlmöglichkeit eröffnen soll. So existieren inzwischen 23 jüdische Jugendzentren in verschiedenen deutschen Städten, die vielfach öffentliche Anerkennung und Förderung erfahren. Verschiedene muslimische Vereine streben derzeit dahin, im Bereich der Jugendverbandsarbeit tätig zu sein und anerkannter Träger der Jugendhilfe bzw. Mitglied in Jugendringen zu werden, was einen hohen Qualifikationsbedarf nach sich zieht. Bei den Kontakten muslimischer Vereine mit kommunalen Einrichtungen stehen die Jugendämter an der Spitze, zu den 37,2 Prozent der Gemeinden Kontakte aufweist; es folgen wenig später Jugendzentren (27,2 Prozent) und Arbeitsgemeinschaften der Jugendhilfe (17,8 Prozent) (Halm / Sauer 2015, 82).

Vielfach geht mit der Entstehung einer islamischen Jugendarbeit eine Verselbständigung junger Muslime von Erwachsenenorganisationen einher. Die Jugendvereine oder -gruppen müssen sich Grundprinzipien wie Freiwilligkeit, Selbstorganisation, Mitverantwortung 
und demokratische Gliederung zu eigen machen, um förderfähig zu werden. Manchen Organisationen wie den Landesjugendverbänden der dem türkischen Staat nahestehenden DITIB (Türkisch-Islamische Union der Anstalt für Religion) gelingt es zudem, religiöse Angebote einerseits und gesellschaftliche, kulturelle wie persönlichkeitsbildende Aktivitäten transparent voneinander zu trennen und so eine Anschlussfähigkeit für staatliche Förderprogramme und ein breites Spektrum an Kooperationspartnern zu erreichen. Da eine solche Trennung nicht immer klar durchgehalten werden kann und religiöse und gesellschaftliche Themen oft eng miteinander verknüpft sind, kann in diesem Fall auch eine staatliche Förderung von Projekten mit religiösen Bezügen gerechtfertigt werden. Durch neue Träger erhöht sich auch die Konkurrenz um Mittel, was zu Konflikten und Abschottungen etablierter Organisationen führen kann. Extremismusprävention erweist sich als ein wichtiger Teilaspekt islamischer Jugendarbeit, wobei damit auch Verdachtsmomente transportiert werden können, die wiederum ein einseitiges Islambild verstärken. Daher bevorzugen verschiedene Projekte, von Empowerment, Identitätsstärkung oder Qualifikationsmaßnahmen zu sprechen und bei einer weit verstandenen Prävention mit Muslimen und ihren Organisationen zusammenzuarbeiten (Hamdan / Schmid 2014, 164ff.).

Sowohl von jüdischen als auch von muslimischen Trägern wird inzwischen eine Reihe von Kindertagesstätten betrieben. Hier ist ebenso wie in anderen Feldern eine Durchmischung wünschenswert, so dass nicht nur jüdische oder muslimische Kinder diese Angebote wahrnehmen. Ein Sonderfall im Bereich der außerschulischen Betreuung sind die Schülerwohnheime des mystisch geprägten und von einer strengen Religionspraxis VIKZ (Verband Islamischer Kulturzentren), die Hausaufgabenhilfe, Freizeitangebote und religiöse Unterweisung miteinander verbinden. In vielen Fällen wurden hier inzwischen mehr oder weniger intensive Kontakte zwischen den Wohnheimen und Schulen aufgebaut, was segregativen Tendenzen entgegenwirkt (Schmid et al. 2008, 166ff.).

Im Bereich der schulischen Bildung kam es in den letzten Jahren vor allem in zweierlei Hinsicht zu Veränderungen: So sind inzwischen 14 meist kleine jüdische Grundschulen entstanden (Ben-Rafael et al. 2011). Sie sind Ausdruck eines erneuerten jüdischen Lebens nach der Shoah und bieten Möglichkeiten einer intensiveren religiösen Erziehung. Daneben gibt es inzwischen 24 Schulen, die von der bildungsorientierten Hizmet-Bewegung um den in den USA lebenden türkischen Prediger Fethullah Gülen (geboren 1938 oder 1941) betrieben werden. Die lokalen Trägervereine der Schulen weisen kein explizit religiöses Profil auf, bieten aber eine besondere Förderung für Kinder und Jugendliche mit Migrationshintergrund. Inwieweit diese Schulen genutzt werden, um junge Menschen auch gezielt für die religiösen Angebote und Kreise der Bewegung anzuwerben, ist umstritten (Hamdan / Schmid 2014, 86f.).

Ein weiteres Feld der Interaktion zwischen Kommunen und religiösen Gemeinden sind Friedhöfe und religiöse Bauten. Jüdische Friedhöfe und Gräberfelder weisen schon eine lange Tradition auf. Da islamische Organisationen nicht als Körperschaften des öffentlichen Rechts anerkannt sind, dürfen sie keine eigenen Friedhöfe einrichten. Inzwischen gibt es jedoch in zahlreichen Kommunen als Teil städtischer Friedhöfe islamische Gräberfelder, in denen die Gräber in Richtung Mekka ausgerichtet sind (Lemmen 2009). Außerdem 
kann in den meisten Bundesländern inzwischen aus religiösen Gründen vom Sargzwang abgesehen werden. Dies ist ein Beispiel dazu, dass Kompromisse und Entgegenkommen möglich sind.

Ebenso wie durch Bestattungen in Deutschland bringen Muslime durch äußerlich erkennbare Moscheebauten zum Ausdruck, dass sie dort dauerhaft ihre Heimat sehen und zudem als kommunale Akteure sichtbar werden wollen. Wo ein Moscheeverein bauen will gibt es sehr oft Proteste von Anwohnern und Widerstand von einem Teil der politischen Verantwortlichen. Oft werden Moscheekonflikte zum brisanten Wahlkampfthema, so dass die Moscheen erst mit großer Verzögerung, an anderer Stelle oder gar nicht gebaut werden. Bei Diskussionen um Moscheebauten, die meist in einer komplexen Konstellation von Akteuren stattfinden (Parteien, Kirchen, kommunale Gremien, Bürgerinitiativen, Gewerkschaften, Medien), wirken rechtspopulistische und islamophobe Akteure konfliktverstärkend (Hohage 2013). Für sie sind Moscheen „Symbole eines neuen, religiös motivierten Kulturkampfes“ (Beinhauer-Köhler / Leggewie 2009, 180). Moscheekonflikte reihen sich in eine Serie von Nachbarschaftskonflikten ein, die auszubrechen pflegen, wo immer in einem Wohngebiet größere Zweckbauten entstehen, die zuerst Baulärm und dann Verkehr, Parkplatznot und andere Erschwernisse nach sich zu ziehen drohen. Es gibt unterschiedliche Strategien auf Seiten von Kommunen im Umgang mit Moscheebauvorhaben: Entweder versuchen sie, Moscheen möglichst an der Peripherie zu verorten und Konflikten durch paternalistische Anordnungen weitgehend aus dem Weg zu gehen. Oder sie versuchen, anhand des Moscheebaus einen von einer möglichst breiten Koalition getragenen Diskussionsprozess zu gestalten, die ansässige Bevölkerung dabei mit einzubeziehen und so die im Konflikt freigesetzte Energie positiv zu nutzen, anstatt Konflikte zu umgehen (vgl. dazu Leggewie et al. 2002, 35f.). Ein vollendeter Moscheebau selbst ist noch kein Garant dafür, dass es zu einer intensiveren Kommunikation kommt. Entscheidend ist, welche Maßnahmen die beteiligten Akteure über den konkreten Konflikt hinaus ergreifen und inwieweit die vorhandene Energie für verschiedene Felder genutzt wird (Jugendarbeit, Seniorenarbeit, Erwachsenenbildung, Beratungsangebote usw.). Der Moscheebaukonflikt kann als Inkorporationsritual verstanden werden (Hüttermann 2006; 2010): Die „Fremden“ werden zuerst abgestoßen, bevor sie in einem kommunalpolitisch bewährten Konfliktprozedere im Hinblick auf Stärken, Absichten und Strategien geprüft werden. Wenn es gelingt, die Auseinandersetzung zu lösen, kehrt wieder Routine ein. Die „Fremden“ sind durch das Konfliktritual zum ersten Mal vernehmbare Akteure der lokalen Öffentlichkeit geworden. Der Moscheeverein kann durch das Ritual lernen, wie wichtig es ist, sich aktiv und transparent in die Stadtgesellschaft einzubringen und vertrauensvolle Beziehungen aufzubauen.

Daneben gibt es weitere, vielfach niederschwellige Interaktionen wie die Nutzung städtischer Räume; Stadtteilfeste sowie die Mitwirkung in kommunalen Gremien. Bestehende Beispiele zeigen, dass die Inkorporation weitgehend gelingen kann. Das System ist dahingehend offen, neue Akteure zu integrieren und bei der Professionalisierung ihrer Arbeit zu unterstützen, ohne dass es dadurch zu einer staatlichen Einmischung in religiöse Inhalte kommt. Die kommunalen Träger und Fachdienste erleben und akzeptieren einen großen 
Teil der islamischen Gemeinden als wertvolle Unterstützer im Integrationsprozess. Es ist auch zu beobachten, dass sich islamische Vereine das Thema Integration selbst zu eigen machen und gewillt sind, entsprechend aktiv zu werden (Aumüller / Gesemann 2014, 123-126). Vielfach geht es zunächst um einen Vertrauensaufbau, der eine Grundlage für das Thematisieren von Konflikten bildet. Die überschaubaren persönlichen Beziehungen sowie die oft hohe Identifikation mit dem Wohnort sprechen für eine „Kommunalisierung der Integrationspolitik“ (Meyer / Schubert 2011, 298). Andererseits zeigen sich auch Grenzen: Aufgrund der weitgehend ehrenamtlich geleisteten Arbeit in islamischen Vereinigungen stehen diesen nur begrenzte Ressourcen für Projekte in sozialen Handlungsfeldern zur Verfügung. So besteht vielfach die Gefahr von Überlastung, Resignation und folgendem Rückzug.

Die Studie „Islamisches Gemeindeleben in Deutschland“ belegt, dass bereits ein „hohe(r) Grad der Vernetzung der Gemeinden mit der deutschen Gesellschaft“ (Halm et al. 2012, 113) besteht. 77 Prozent der befragten Gemeinden geben an, über Kontakte und Kooperationen mit Kirchengemeinden zu verfügen; es folgen die Polizei mit 71 Prozent und bereits an dritter Stelle die Schulen mit 68 Prozent. Aus kommunaler Sicht stellt sich angesichts der vielfältigen islamischen Organisationsstrukturen die Frage, mit welchen Vereinen eine Zusammenarbeit stattfinden soll. Konflikte ergeben sich insbesondere hinsichtlich der Einbindung der vom Verfassungsschutz beobachteten Vereine. Es geht dabei vor allem um die zunächst in einem türkisch-nationalen Islamismus verwurzelte IGMG (Islamische Gemeinschaft Milli Görüş), die in den letzten Jahren einen starken Wandel hin zu einem „Postislamismus“ durchlaufen hat (Schiffauer 2010) und inzwischen nur noch in manchen Bundesländern beobachtet wird. Aufgrund der Vielfalt islamischer Vereine, die besteht, auch wenn diese demselben Dachverband angehören, ist die kommunale Urteils- und Einschätzungskompetenz von zentraler Bedeutung. Oft ist es so, dass verschiedene Gemeinden unterschiedlich stark am kommunalen Leben beteiligt sind und auch in unterschiedlicher Weise von den Kommunen einbezogen werden. So gibt es das Phänomen von „Vorzeigemoscheen“, die privilegierte Partner der Kommunen sind (Schmid et al. 2008, 230). Kehrseite solcher Entwicklungen ist, dass andere Vereine nicht dieselbe Förderung erfahren. Aus integrationspolitischer Sicht ist eine derartige Ungleichbehandlung problematisch. Somit steht die kommunale Integrationspolitik vor der Herausforderung, sich mit den lokalen muslimischen Organisationsstrukturen in ihrer Vielfalt auseinanderzusetzen.

Veränderungsprozesse nicht nur im Fall der IGMG, sondern auch innerhalb der meisten anderen islamischen Gruppen und Organisationen haben schließlich weitreichende Auswirkungen auf Konflikte. Durch den Generationenwandel und Bildungsaufstieg von wichtigen Personen innerhalb der Gemeinden sind die sprachlichen und kommunikativen Kompetenzen und damit auch die Konfliktfähigkeit und -bereitschaft vielerorts gestiegen. Jedoch stehen gerade die Imame angesichts der hohen Anforderungen an sie nicht nur als religiöse Experten, sondern auch als Integrationslotsen vor sehr großen Herausforderungen (Halm et al. 2012, 333-389). Ein wichtiger Faktor sind Qualifizierungsmaßnahmen auch innerhalb islamischer Organisationen (wie das von DITIB durchgeführte Projekt „proDialog“ [Rosenow-Williams 2012, 187-248) und eine gezielte Förderung auf kommunaler 
Ebene (wie etwa in den Projekten „MünchenKompetenz“ oder „Muslimische Gemeinden als kommunale Akteure“). Solche Schritte führen nicht nur zu einer Professionalisierung, sondern auch zu einem gestiegenen Selbstbewusstsein, mit welchem muslimische Akteure den Kommunen gegenüber treten.

Gerade das Feld islamischer Akteure macht deutlich, dass Religion als Faktor wieder in die Debatte zurückkehrt und damit auch Prämissen wie die Trennung von Religion und sozialer Arbeit hinterfragt werden. So geht es insbesondere darum, inwieweit es Kommunen gelingt, gesprächsfähig zu sein und sich religiöse Trends zunutze zu machen. Die verbreitete Aufgeschlossenheit islamischer Vereinigungen kann genutzt werden, um sie in Kommunen auf möglichst vielen Ebenen einzubinden.

Die massenhafte Einwanderung von Muslimen (Syrien, Afghanistan, Pakistan, Albanien und Kosovo waren Hauptherkunftsstaaten in den Jahren 2014 und 2015) stellt hier eine neue Herausforderung dar, die aber auch die große Chance bietet, Schulen, Sozialeinrichtungen, Heime und andere Orte zu vernetzen und damit auch den interreligiösen Dialog und die Kooperation zu fördern. Damit können Vereinseitigungen konterkariert werden, die in einigen überwiegend von Salafisten beherrschten Moscheegemeinden zu beobachten waren. Eine Orientierung, wie ein aufrichtiger und symmetrischer Dialog gestaltet werden könnte, hat der Islamwissenschaftler und Schriftsteller Navid Kermani bei einer Solidaritätsdemonstration für Charlie Hebdo in Köln gegeben, als er sagte: „In dem Augenblick, da sich Terroristen auf den Islam berufen, hat der Terror auch etwas mit dem Islam zu tun. Wir müssen die Auseinandersetzung mit der Lehre suchen, die heute weltweit Menschen gegeneinander aufhetzt und Andersgläubige ermordet oder erniedrigt“. Kermani appellierte an „meine Geschwister im Glauben“, ihre staatsbürgerliche Pflicht ernst zu nehmen und für Freiheit und Gerechtigkeit einzutreten, indem er sie an eine Säule ihrer Religion erinnert: „Vor allem aber liegt es an uns, dem höchsten Gebot des Islams, der Barmherzigkeit, wieder Geltung zu verschaffen. ,Wahrlich, erhebst du auch deine Hand gegen mich, um mich totzuschlagen, so erhebe ich doch nicht meine Hand gegen dich, um dich zu erschlagen' - das werden heute die meisten für die Bergpredigt halten, ist aber doch unserer eigener Koran, Sure 5,28.“ (Frankfurter Allgemeine Zeitung, 16.01.2015)

\section{$5 \quad$ Politische und interreligiöse Dialoge als Wege der Konfliktmoderation}

Symbolische Konflikte, die religiöse Gefühle und Überzeugungen betreffen, scheinen unteilbar zu sein und damit oft auch unlösbar. Auf unterschiedlichen Ebenen und in unterschiedlichen Feldern wurden jedoch mehr oder weniger erfolgreich Versuche gestartet, Konflikte zu deeskalieren und Lösungsstrategien zu entwickeln. Diese Versuche sind oftmals vom Leitbild des Dialogs als eines verständigungsorientierten Prozesses geprägt. Auch wenn damit noch keine rechtliche Anerkennung erreicht wird, so doch „soziale Wertschätzung“, 
die dafür eine Vorstufe darstellt (Honneth 1993, 179f.). Die Dialog- und Interaktionsprozesse fungieren ferner als Gegenbild zu einseitig negativen Islamdarstellungen.

Dialoge sind gesellschaftliche Kommunikationsprozesse, die auf bestimmten sozialen und politischen Ausgangslagen beruhen (Dehn 2014). Diese sind vielfach von Asymmetrien hinsichtlich Macht und Einfluss, sozialer und ökonomischer Situation, Bildungsstand sowie Interessen der beteiligten Akteure geprägt (Schmid 2010a). Dass sich Dialogpartner auf gleicher Ebene begegnen, ist ein selten erreichtes Ideal. Dies muss bei der Gestaltung von Dialogprozessen berücksichtigt werden, denen auch die Aufgabe zukommt, auch die jeweilige konkrete soziale Ausgangslage zu reflektieren und zu berücksichtigen. Dialog ist kein Selbstzweck, sondern bestimmten Zielen verpflichtet. Diese werden im Idealfall nicht von einer Seite gesetzt, sondern gemeinsam bestimmt, damit der Dialog mehr ist als eine inszenierte Alibi- oder Schauveranstaltung. Die Beispiele von unterschiedlichen Ebenen illustrieren, dass Dialog nicht immer gelingt, aber trotz bestehender Schwierigkeiten einiges erreicht hat. Wichtig ist die Ergebnisoffenheit des Dialogs. Dies enthebt aber nicht von der Aufgabe, darauf zu schauen, welche Wirkungen er auf der individuellen, institutionellen, gesellschaftlichen oder medialen Ebene aufweist. Aufgrund der im Dialog angestrebten Verständigung entsteht manchmal das Missverständnis, dass der Dialog an sich harmonisierend sei. Auch wenn manche Dialoge diese Tendenz haben mögen, schließt Dialog gerade das Ringen um einen friedlichen und klärenden Umgang mit Differenzen ein. In der Praxis können Dialoge ganz unterschiedlich ausfallen, da es kein einheitliches Konzept, sondern verschiedene philosophische, kommunikationstheoretische, politische und auch religiös-weltanschauliche Zugänge gibt, um die herum der Begriff Dialog eine lose Klammer bildet. So hängt das Gelingen von Dialogen in der Regel von den beteiligten Personen und Institutionen und ihrer Veränderungsbereitschaft ab.

Auf Bundesebene ist die seit 2006 bestehende Deutsche Islam Konferenz (DIK) zu nennen, mit der erstmals ein deutschlandweiter Dialogprozess stattfindet und an der seit 2014 zehn islamische Dachverbände teilnehmen. Sie ist ein Dialogforum zwischen Vertretern des deutschen Staates und Muslimen in Deutschland. An ihr nehmen aber nicht nur Vertreter des Bundes, sondern auch der Länder und Kommunen teil. Die DIK hat mehrere Studien in Auftrag gegeben, Empfehlungen verfasst und Projekte angestoßen. So wurde hier etwa ein Leitfaden für die Fortbildung von religiösem Personal aus islamischen Gemeinden auf kommunaler Ebene erarbeitet (Geschäftsstelle der Deutschen Islam Konferenz / Bundesamt für Migration und Flüchtlinge 2011). Für die Kommunen kann die DIK eine Vorbildwirkung etwa hinsichtlich der Breite muslimischer Mitwirkender geben und gerade im Fall von Kontroversen ein inhaltlicher wie symbolischer Referenzpunkt mit einer gewissen Autorität und Vorbildwirkung sein.

Auf Länderebene finden teilweise vergleichbare Dialoge und runde Tische statt. In den Stadtstaaten Hamburg und Bremen wurden bereits Staatsverträge mit islamischen Dachverbänden abgeschlossen - in Niedersachsen wird derzeit noch verhandelt. Sie halten den Rechtsstand zu Themen wie Feiertage, Religionsunterricht, islamische Theologie, Seelsorge in Gefängnissen, Moscheebauten etc. fest. An vielen Stellen enthalten sie Willensbekundungen und stellen ein wichtiges politisches Signal dar. Durch sie hat sich Handlungssicherheit 
für Muslime und Verwaltungsmitarbeitende wesentlich erhöht. Außerdem wurde erreicht, dass Ermessensspielräume auf kommunaler Ebene verstärkt zugunsten wechselseitiger Dialogprozesse genutzt werden. Ein Beispiel dafür ist die Öffnung von Stadtteilschulen in Hamburg für muttersprachlichen Unterricht der Moscheegemeinden.

Auch auf kommunaler Ebene gibt es eine Fülle von runden Tischen, Ausländer- oder Integrationsbeiräten. Sie unterscheiden sich in ihrer Struktur, Intensität und in ihrer thematischen Ausrichtung. Auf diese Weise entstehen vor Ort Netzwerke unterschiedlicher staatlicher wie zivilgesellschaftlicher Akteure und Foren, in denen auch das Zusammenspiel von Fragen der Religion und des Zusammenlebens behandelt werden können. Daneben sind aber gleichermaßen unterschiedliche Fachdienste und Behörden vom Sozial- und Jugendamt über das Schulverwaltungsamt bis zum Bau- und Friedhofsamt betroffen (Kommunaler Qualitätszirkel zur Integrationspolitik 2012 [mit zahlreichen Beispielen und Empfehlungen]). Den kommunalen Integrationsbeauftragten kommt eine wichtige moderierende Funktion in diesem vielfältigen Gefüge zu (Gesemann et al. 2012, 151).

Ein weiterer Faktor ist der interreligiöse Dialog, der ein breites Spektrum an Zugängen und Handlungsfeldern aufweist (Meißner et al. 2014). Ferner gibt es eine Vielfalt unterschiedlicher Träger und Organisationsformen. Zudem erfüllt der Dialog unterschiedliche Funktionen: Während er für Christen oft mit Bildung oder diakonischem Engagement verbunden ist, erweist er sich für Muslime als Schritt hin zu Partizipation und Ankommen in der Gesellschaft. Dialog ist in hohem Maße abhängig von Personen und institutionellen Konstellationen sowie Offenheit der Kommune, mit religiösen Akteuren zusammenzuarbeiten. So verstehen sich manche Kommunen als Organisatoren und Moderatoren des interreligiösen Dialogs, während sich andere aus Neutralitätsgründen strikt zurückhalten. Spiegelbildlich dazu gibt es Dialogakteure, die keinen Einfluss auf das lokale Integrationsgeschehen nehmen wollen, da der Dialog für sie auf rein religiöse Themen beschränkt bleiben soll. Vielfach wird der Dialog jedoch auch ganzheitlich verstanden als „Dialog des Handelns" unter Einbeziehung von Fragen des Zusammenlebens in einer pluralistischen Gesellschaft, die positiv wahrgenommen wird (Zentralkomitee der deutschen Katholiken 2012). Dann besteht die Möglichkeit, dass sich religiöse Akteure auch an kommunalen Foren, Gremien oder runden Tischen beteiligen und so zum Ausdruck bringen, dass sie sich nicht in einer religiösen Sonderwelt verorten, sondern sich als Teil der Zivilgesellschaft verstehen und über ihre eigene Gemeinde hinaus Mitverantwortung für das Ganze tragen. Unterschiedliche sozialpolitische Themen wie Arbeit, Wohnen, Bildung und Beteiligung können so auch im Grenzbereich von staatlicher Rahmengebung und zivilgesellschaftlichem Engagement angegangen werden (Mund / Theobald 2009, 190-266).

Im Konfliktfall zwischen einer Kommune und Muslimen kann es dazu kommen, dass eine Moderation oder Mediation durch angesehene Persönlichkeiten oder Institutionen aus der Kommune oder durch externe Berater erforderlich wird. Da die Kirchen eine besondere Sensibilität und Sympathie für religiöse Belange haben, genießen sie oft das Vertrauen der muslimischen Seite. Umgekehrt werden sie von staatlicher bzw. kommunaler Seite als etablierte Akteure ernst genommen und können so eine Mittlerposition einnehmen. Den Kirchen wird von verschiedenen Seiten zugetraut, unabhängig und relativ frei von 
Eigeninteressen zu sein. So können sie in Konflikten eine Schlüsselrolle spielen. Indem Muslime zunehmend einen direkten Zugang zu staatlichen Stellen finden, verliert diese vermittelnde Funktion des interreligiösen Dialogs an Bedeutung und er verortet sich weniger in Ausrichtung auf den Staat denn als Teil der Zivilgesellschaft. Der interreligiöse Dialog kann in unterschiedlicher Weise auf Konflikte einwirken, die selbst schon eine Beziehung zwischen unterschiedlichen Gruppen herstellen. So kann er Konflikte verstärken, indem religiöse Zuschreibungen und Aufladungen ein hohes Gewicht bekommen, indem stärker normativ statt pragmatisch argumentiert und eine Allianz der Gläubigen gegen die Areligiösen angestrebt wird, die dem Gedanken der Partizipation entgegensteht. Es kann durch den Dialog zu typisierenden Zuschreibungen kommen, die der realen Vielfalt innerhalb der Religionen und auch innerhalb der lokalen Gemeinden entgegensteht. Umgekehrt kann sich der interreligiöse Dialog als Instrument des Konfliktmanagements erweisen (Schmid 2010b), indem ein differenzierender und konstruktiver Umgang mit Unterschieden erfolgt, die im Ergebnis nicht mehr als bedrohlich, sondern als komplementär angesehen werden. Exklusive Ansprüche können dabei in einer inklusiven Synthese auf einer höheren Ebene überwunden werden. Darüber hinaus können die konkrete Begegnung und Vernetzung durch den Dialog erleichtert und Kompromisse erarbeitet werden. Auf diese Weise wird der Konflikt lokalisiert, anstatt ihn zu globalisieren, was ihn überschaubar und gestaltbar macht. Zudem werden im Dialog auch politik- und ideologiekritische Positionen eingenommen. Es kommt so gerade nicht zu einer Verdrängung des Konflikts, sondern zu dessen institutionalisierter Bearbeitung in der Form des Dialogs. Laut der bisher größten quantitativen und qualitativen Studie zum christlich-islamischen Dialog in Deutschland kann dieser als „Forum kooperativer Problembearbeitung“ (Klinkhammer et al. 2011, 291) verstanden werden, wobei wie die Studie zeigt, dass Konfliktlinien oft nicht zwischen den Religionen verlaufen, sondern oft quer zu ihnen, so dass eine größere Nähe zwischen verschiedenen Gruppen aus unterschiedlichen Religionen besteht als zwischen Angehörigen einer Religion. Ob es gelingt, konstruktiv mit Konflikten umzugehen, oder ob doch die konfliktverstärkenden und -eskalierenden Aspekte mehr zur Geltung kommen, hängt von der konkreten Gestaltung des Dialogs ab. Dies erfordert kommunikative, interkulturelle und interreligiöse Kompetenzen auf Seiten der beteiligten Akteure. Die Wirkmöglichkeiten des interreligiösen Dialogs dürfen auch nicht überschätzt werden. So ist der Einfluss der Religionsgemeinschaften begrenzt, und oftmals stellt der Dialog keine wirkliche Priorität innerhalb ihrer unterschiedlichen Handlungsfelder dar.

Im positiven Fall mag es gelingen, dass sich die verschiedenen Ebenen vom Bund bis zur Kommune sowie politischer wie interreligiöser Dialog wechselseitig ergänzen und inspirieren. Ebenso können Impulse von außen und innere Öffnungsprozesse in islamischen Organisationen einander verstärken und zu einer besseren Integration beitragen (Schmid et al. 2008, 198ff.). Dialog erweist sich als ein Ansatz, der sowohl auf den verschiedenen formellen und institutionellen Ebenen als auch in anderen Bereichen der Interaktion zur Geltung kommen kann. So kann beispielweise eine intensive Kommunikation zwischen Lehrkräften und muslimischen Eltern Misstrauen abbauen und Kompromisse hervorbringen, indem ein geschlechtergetrennter Schwimm- und Sportunterricht durchgeführt wird, 
Schulen muslimische Feiertage in ihrer Terminplanung berücksichtigen und Klassenfahrten von einem muslimischen Elternteil oder einer Vertrauensperson begleitet werden (Hinrichs et al. 2012). Dies schließt allerdings nicht aus, dass bei manchen Themen wie Sexualerziehung im Sinne des schulischen Bildungsauftrags klare Grenzlinien gezogen werden.

\section{$6 \quad$ Fazit}

In diesem Beitrag haben wir zu zeigen versucht, wie lokale Politik durch globale Religionskonflikte und transnationale Religionspolitik überlagert wird, aber auch durch das gesellschaftliche Engagement religiöser Gemeinden vor Ort mitgeprägt wird. Häufig steht beides in einem Spannungsverhältnis, was einen Ausgangspunkt für die politische Gestaltung des Zusammenlebens bildet. Eine Weiterentwicklung des Staat-Kirche-Regimes im Blick auf neue Akteure, insbesondere Muslime, kann ein wichtiger Schritt hin zu Integration und Partizipation sein. Auf lokaler Ebene bietet etwa der Bereich der Kinder- und Jugendhilfe ein Feld, in dem in einem staatlich gesetzten Rahmen und in einem breiten Spektrum an Organisationen auch verschiedene religiöse Akteure tätig werden können, was für alle Beteiligte ein Lernfeld im Umgang mit Vielfalt darstellt. Dabei muss den organisationsspezifischen Besonderheiten des Islams und insbesondere der innermuslimischen Pluralität Rechnung getragen werden. Auf Seiten der Religionen, der Verwaltung und der Kommunalpolitik sind in wachsendem Maße Kompetenzen erforderlich, um mit dieser neuen und in hohem Maße komplexen Situation umzugehen. Einübung in Konflikt- und Dialogfähigkeit, Moderation und Prävention spielen dabei eine zentrale Rolle. Kommunale Verwaltungen können sich auch auf zivilgesellschaftliche Akteure stützen, die bereits über intensive Erfahrungen mit Muslimen verfügen. In jedem Fall geht es darum, das sehr dynamische Feld islamischer Gruppen und Organisationen nicht sich selbst zu überlassen, sondern es zunächst differenziert wahrzunehmen, es sodann gezielt zu fördern und partnerschaftlich einzubeziehen.

\section{Literatur}

Aumüller, Jutta / Gesemann, Frank 2014: Abschlussbericht Forschungs-Praxis-Projekt: Integrationspotenziale ländlicher Regionen im Strukturwandel. Darmstadt: Schader-Stiftung

Beinhauer-Köhler, Bärbel / Leggewie, Claus 2009: Moscheen in Deutschland. Religiöse Heimat und gesellschaftliche Herausforderung. München: C.H. Beck

Ben Rafael, Eliezer / Glöckner, Olfa / Sternberg, Yitzhak 2011: Jews and Jewish Education in Germany Today. Leiden: Brill

Benz, Wolfgang 2011: Antisemitismus und „Islamkritik“. Bilanz und Perspektiven. Berlin: Metropol Bertelsmann Stiftung (Hrsg.) 2007: Religionsmonitor 2008. Gütersloh: Gütersloher Verlagshaus 
Bielefeldt, Heiner 2007: Menschenrechte in der Einwanderungsgesellschaft. Plädoyer für einen aufgeklärten Multikulturalismus. Bielefeld: transcript

Bielefeldt, Heiner (2008): Das Islambild in Deutschland. Zum öffentlichen Umgang mit der Angst vor dem Islam. Berlin: Deutsches Institut für Menschenrecht

Brettfeld, Karin / Wetzels, Peter 2007: Muslime in Deutschland. Integration, Integrationsbarrieren, Religion und Einstellungen zu Demokratie, Rechtsstaat und politisch-religiös motivierter Gewalt. Berlin: Bundesministerium des Innern

Çakır, Naime 2014: Islamfeindlichkeit. Anatomie eines Feindbildes in Deutschland. Bielefeld: transcript

Dehn, Ulrich 2014: Der christlich-islamische Dialog auf dem Hintergrund gesamtgesellschaftlicher Veränderungen. In: Rohe, Mathias et al. (Hrsg.) 2014: Handbuch Christentum und Islam in Deutschland, Freiburg i. Br.: Herder, Bd. 2, 1011-1038

Eißler, Friedmann 2009: Muslimische Einladung zum Dialog. Dokumentation zum Brief der 138 Gelehrten („A Common Word“). Berlin: Evangelische Zentralstelle für Weltanschauungsfragen

Färber, Alexa / Spielhaus, Riem (Hrsg.) 2006: Islamisches Gemeindeleben in Berlin. Berlin: Der Beauftragte des Senats von Berlin für Integration und Migration

Forst, Rainer 2003, Toleranz im Konflikt. Geschichte, Gehalt und Gegenwart eines umstrittenen Begriffs. Frankfurt a. M.: Suhrkamp

Geschäftsstelle der Deutschen Islam Konferenz / Bundesamt für Migration und Flüchtlinge 2011, Dialog Öffnung Vernetzung. Leitfaden für die gesellschaftskundliche und sprachliche Fortbildung von religiösem Personal und weiteren Multiplikatoren islamischer Gemeinden auf kommunaler Ebene. Nürnberg

Gesemann, Frank / Roth, Roland / Aumüller, Jutta 2012: Stand der kommunalen Integrationspolitik in Deutschland. Berlin: Beauftragte der Bundesregierung für Migration, Flüchtlinge und Integration/Bundesministerium für Verkehr, Bau und Stadtentwicklung

Hafez, Kai / Schmidt, Sabrina 2015: Die Wahrnehmung des Islams in Deutschland. Religionsmonitor - verstehen was verbindet. Sonderauswertung Islam 2015. Gütersloh: Bertelsmann Stiftung

Hamdan, Hussein / Schmid, Hansjörg 2014: Junge Muslime als Partner. Ein empiriebasierter Kompass für die soziale Arbeit. Weinheim: Beltz Juventa

Halm, Dirk / Sauer, Martina / Schmidt, Jana/ Stichs, Anja 2012: Islamisches Gemeindeleben in Deutschland. Im Auftrag der Deutschen Islamkonferenz. Nürnberg: Bundesamt für Migration und Flüchtlinge

Halm, Dirk / Sauer, Martina 2015: Soziale Dienstleistungen der in der Deutschen Islam Konferenz vertretenen religiösen Dachverbände und ihrer Gemeinden. Studie im Auftrag der Deutschen Islamkonferenz. Berlin: Bundesministerium des Innern

Hasenclever, Andreas 2008: Merkmale gewaltresistenter Glaubensgemeinschaften - Überlegungen zum Schutz religiöser Überlieferung vor politischer Vereinnahmung. In: Brocker, Manfred / Hildebrandt, Mathias (Hrsg.), Friedensstiftende Religionen? Religion und die Deeskalation politischer Konflikte. Wiesbaden: Springer VS, S. 179-201

Haug, Sonja / Müssig, Stephanie / Stichs, Anja 2009: Muslimisches Leben in Deutschland. Im Auftrag der Deutschen Islam Konferenz. Nürnberg: Bundesamt für Migration und Flüchtlinge

Hero, Markus / Krech, Volkhard / Zander, Helmut (Hrsg.) 2008: Religiöse Vielfalt in Nordrhein-Westfalen. Empirische Befunde und Perspektiven der Globalisierung vor Ort. Paderborn: Schöningh Hinrichs, Ulrike / Romdhane, Nizar / Tiedemann, Markus 2012: „Unsere Tochter nimmt nicht am Schwimmunterricht teil!" 50 religiös-kulturelle Konfliktfälle in der Schule und wie man ihnen begegnet. Mülheim an der Ruhr: Verlag an der Ruhr

Hohage, Christoph 2013: Moschee-Konflikte. Wie überzeugungsbasierte Koalitionen lokale Integrationspolitik bestimmen. Wiesbaden: Springer VS 
Honneth, Axel 1993: Kampf um Anerkennung. Zur moralischen Grammatik sozialer Konflikte. Frankfurt a. M.: Suhrkamp

Huber, Stefan 2007: Aufbau und strukturierende Prinzipien des Religionsmonitors. In: Bertelsmann Stiftung 2007, S. 19-24

Hüttermann, Jörg 2006: Das Minarett. Zur politischen Kultur des Konflikts um islamische Symbole. Weinheim/München: Beltz Juventa

Hüttermann, Jörg 2010: Entzündungsfähige Konfliktkonstellationen: Eskalations- und Integrationspotenziale in Kleinstädten der Einwanderungsgesellschaft. Weinheim / München: Juventa

Kermani, Navid 2009: Wer ist Wir? Deutschland und seine Muslime. München: C. H. Beck

Klinkhammer, Gritt / Frese, Hans-Ludwig / Satilmis, Ayla / Seibert, Tina 2011: Interreligiöse und interkulturelle Dialoge mit Muslimen in Deutschland. Eine quantitative und qualitative Studie. Bremen: Universität Bremen

Klöcker, Michael / Tworuschka, Udo (1997ff.): Handbuch der Religionen: Kirchen und andere Glaubensgemeinschaften in Deutschland. Landsberg am Lech: Olzog

Kommunaler Qualitätszirkel zur Integrationspolitik 2012, „Umgang mit religiöser Vielfalt - Handreichung für die kommunale Praxis“. Stuttgart

Krech, Volkhard 2007: Exklusivität, Bricolage und Dialogbereitschaft. Wie die Deutschen mit religiöser Vielfalt umgehen. In: Bertelsmann Stiftung 2007, Religionsmonitor 2008. Gütersloh: Gütersloher Verlagshaus, S.33-43

Krech, Volkhard 2008: Bewegungen im religiösen Feld: Das Beispiel Nordrhein-Westfalen. In: Hero et al. 2008, Religiöse Vielfalt in Nordrhein-Westfalen. Empirische Befunde und Perspektiven der Globalisierung vor Ort. Paderborn: Schöningh, S. 24-43

Leggewie, Claus 1993: Alhambra. Der Islam im Westen. Reinbek bei Hamburg: Rowohlt

Leggewie, Claus 1996: How Turks Became Kurds, Not Germans. In: Dissent 43/3. 79-83

Leggewie, Claus 2009: Weltmacht Religion. In: Debiel, Tobias et al. (Hrsg.): Globale Trends 2010. Frieden, Entwicklung, Umwelt. Frankfurt a. M.: Fischer, S. 61-80

Leggewie, Claus / Joost, Angela / Rech, Stefan (Hrsg.) 2002: Der Weg zur Moschee. Eine Handreichung für die Praxis. Bad Homburg: Herbert-Quandt-Stiftung

Lemmen, Thomas 2009: Angebot und Nachfrage - Muslimische Grabfelder in Deutschland. In: Reiner Sörries (Hrsg.): Muslime in deutscher Erde. Sterben, Jenseitserwartung und Bestattung. Kassel: Arbeitsgemeinschaft Friedhof und Denkmal, S. 55-62

Meißner, Volker /Affolderbach, Martin / Mohagheghi, Hamideh / Renz, Andreas (Hrsg.) 2014: Handbuch christlich-islamischer Dialog. Grundlagen - Themen - Praxis - Akteure. Freiburg i. Br.: Herder

Meyer, Hendrik / Schubert, Klaus 2011: Vielfalt als Potential - Implikationen aus dem Verhältnis von Politik und Islam. In: dies. (Hrsg.): Politik und Islam. Wiesbaden: VS Verlag für Sozialwissenschaften, S. 290-310

Müller, Harald 1998: Das Zusammenleben der Kulturen. Ein Gegenentwurf zu Huntington. Frankfurt a. M.: Fischer

Mund, Petra / Theobald, Bernhard 2009 (Hrsg.): Kommunale Integration von Menschen mit Migrationshintergrund - ein Handbuch. Berlin: Deutscher Verein für öffentliche und private Fürsorge

Norris, Pippa / Inglehart, Ronald 2011: Sacred and Secular. Religion and Politics Worldwide. 2nd Edition. Cambridge: Cambridge University Press

Pollack, Detlef / Müller, Olaf 2013: Religionsmonitor. verstehen was verbindet. Religiosität und Zusammenhalt in Deutschland. Gütersloh: Bertelsmann Stiftung (https://www.bertelsmann-stiftung.de/fileadmin/files/BSt/Publikationen/GrauePublikationen/GP_Religionsmonitor_verstehen_was_verbindet_Religioesitaet_und_Zusammenhalt_in_Deutschland.pdf [31.8.2015]) 
Rohe, Mathias / Engin, Havva / Khorchide, Mouhanad / Özsoy, Ömer / Schmid, Hansjörg (Hrsg.) 2014: Handbuch Christentum und Islam in Deutschland. Grundlagen, Erfahrungen und Perspektiven des Zusammenlebens. 2 Bde. Freiburg i. Br.: Herder

Rosenow-Williams, Kerstin 2012: Organizing Muslims and Integrating Islam in Germany. New Developments in the 21st Century. Leiden/Boston: Brill

Schiffauer, Werner 2010: Nach dem Islamismus. Eine Ethnographie der Islamischen Glaubensgemeinschaft Milli Görüş. Frankfurt a. M.: Suhrkamp

Schmid, Hansjörg (2010a): Zwischen Asymmetrie und Augenhöhe. Zum Stand des christlich-islamischen Dialogs in Deutschland. In: Hünseler, Peter / Di Noia, Salvatore (Hrsg.): Kirche und Islam im Dialog. Eine europäische Perspektive. Regensburg: Pustet, S. 49-89

Schmid, Hansjörg (2010b): Integration durch interreligiösen Dialog? Versuch einer Verhältnisbestimmung, in: Ucar, Bülent (Hrsg.): Die Rolle der Religion im Integrationsprozess. Die deutsche Integrationsdebatte. Frankfurt a. M.: Peter Lang, S. 519-538

Schmid, Hansjörg 2014: Christen und Muslime als Träger sozialer Verantwortung und Mitgestalter der deutschen Gesellschaft - aus christlicher Perspektive. In: Rohe, Mathias et al. 2014: Handbuch Christentum und Islam in Deutschland. Grundlagen, Erfahrungen und Perspektiven des Zusammenlebens. Freiburg i. Br.: Herder, Bd. 2, S. 627-663

Schmid, Hansjörg / Akca, Ayşe Almıla / Barwig, Klaus 2008: Gesellschaft gemeinsam gestalten. Islamische Vereinigungen als Partner in Baden-Württemberg. Baden-Baden: Nomos

Schreiner, Stefan 2008: Das „christliche Europa“ - eine Fiktion. In: Miksch, Jürgen (Hrsg.): Vom Christlichen Abendland zum abrahamischen Europa. Frankfurt a. M.: Lembeck, S. 126-144

Soysal, Yasemin Nuhoğlu 1994: Limits of Citizenship. Migrants and Postnational Membership in Europe. Chicago: University Press

Willems, Ulrich 2003: Religion als Privatsache? Eine kritische Auseinandersetzung mit dem liberalen Prinzip einer strikten Trennung von Politik und Religion. In: Minkenberg, Michael / Willems, Ulrich (Hrsg.): Politik und Religion. Politische Vierteljahresschrift. Sonderheft 33. Wiesbaden: Westdeutscher Verlag, S. 88-112

Zentralkomitee der deutschen Katholiken (Hrsg.) 2012: Christen und Muslime - Partner in der pluralistischen Gesellschaft. Eine gemeinsame Auseinandersetzung mit gesellschaftlichen Fragen, Bonn (www.zdk.de/veroeffentlichungen/erklaerungen/detail/Christen-und-Muslime-Partner-in-der-pluralistischen-Gesellschaft-Eine-gemeinsame-Auseinandersetzung-mit-gesellschaftlichen-Fragen-208q/ [31.8.2015]) 